04

\title{
Перспектива использования кремниевых фотоумножителей в детекторной камере малоразмерного черенковского гамма-телескопа для снижения порога регистрации космического излучения
}

\author{
() Е.Е. Холупенко, А.М. Красильщиков, Д.В. Бадмаев, А.А. Богданов, Ю.В. Тубольцев, Ю.В. Чичагов, \\ А.С. Антонов, Д.О. Кулешов, Е.М. Хилькевич
}

Физико-технический институт им. А.Ф. Иоффре РАН, 194021 Санкт-Петербург, Россия

e-mail: eugene@astro.ioffe.ru

Поступило в Редакцию 24 октября 2019 г.

В окончательной редакции 23 декабря 2019 г.

Принята к публикации 13 января 2020 г.

\begin{abstract}
Представлены результаты моделирования полезного и шумового сигналов в новой камере черенковского гамма-телескопа TAIGA-IACT, основанной на кремниевых фотоумножителях (SiPM). Показано, что применение современных кремниевых фотоумножителей в качестве детектирующего элемента TAIGA-IACT вместо ныне используемых традиционных вакуумных фотоумножителей позволит снизить пороговую энергию регистрации космических гамма-квантов примерно в 2.5 раза: с $\simeq 1.5$ до $\simeq 0.6 \mathrm{TeV}$. Также показано, что применение в камере TAIGA-IACT стандартной ультрафиолетовой маски-фильтра типа ZWB3 приведет к снижению среднего уровня полезного сигнала примерно в 3 раза и снижению уровня шумового (фонового) сигнала от ночного неба примерно в 6 раз, что позволит увеличить рабочий цикл телескопа (в частности, вести наблюдения в лунные ночи и в сумерках) и даст возможность дополнительно снизить пороговую энергию регистрации до $\simeq 0.3 \mathrm{TeV}$. Применение более узкого УФ фильтра с полосой пропускания 260-300 nm может повысить эффективность определения типа первичной частицы (гамма-адронной сепарации) в диапазоне энергий от $\sim 25$ до $\sim 50 \mathrm{TeV}$.
\end{abstract}

Ключевые слова: широкие атмосферные ливни, гамма-адронная сепарация (гамма-адронный отбор), черенковские гамма-телескопы, детекторы ультрафиолетового излучения.

DOI: $10.21883 /$ JTF.2020.06.49278.354-19

\section{Введение}

Черенковские гамма-телескопы - это инструменты, осуществляющие наблюдение космических источников гамма-излучения посредством регистрации черенковского излучения электронов и позитронов широких атмосферных ливней (ШАЛ), инициированных первичными гамма-квантами при взаимодействии с атмосферой Земли ${ }^{1}$. В камерах современных черенковских гамма-телескопов (MAGIC [1], VERITAS [2], H.E.S.S. [3]) регистрация вспышек черенковского излучения ШАЛ осуществляется с помощью высоковольтных вакуумных фотоумножителей (ФЭУ) с чувствительностью преимущественно в диапазоне длин волн $300-600 \mathrm{~nm}$, что существенно ограничивает периоды времени, в которые возможно осуществлять наблюдения ввиду того, что оптическая засветка от Луны и в сумеречное время от атмосферы приводит к полному ослеплению вакуумных ФЭУ и более того может полностью вывести их из строя. Таким образом, годовая экспозиция этих телескопов составляет порядка $1000 \mathrm{~h}$. Наиболее вероятно, что в детекторных блоках разрабатываемых в настоящее время телескопов нового (четвертого) поколения

\footnotetext{
1 Далее в тексте под термином „электроны ШАЛ“ будут пониматься вторичные электроны и позитроны ШАЛ.
}

(например, CTA [4], ALEGRO [5]) будут использованы полупроводниковые фотодетекторы - кремниевые фотоумножители (SiPM). Применение камеры на основе SiPM в телескопе-прототипе FACT уже сейчас позволяет проводить наблюдения даже в лунные ночи (посредством всего лишь увеличения порога регистрации сигнала в камере), что существенно увеличивает экспозиции исследуемых космических источников [6]. Дополнительным методом, позволяющим значительно увеличить рабочий цикл черенковского гамма-телескопа, является использование УФ-фильтров [7-10], блокирующих излучение оптического диапазона и пропускающих УФ-излучение, что приводит к значительному подавлению шумового сигнала в камере телескопа (вызванного оптическим фоном ночного неба и лунной засветкой) при сохранении значительной части полезного сигнала, поскольку спектр черенковского излучения ШАЛ имеет максимум интенсивности на длинах волн 300-330 nm.

Еще одной важной возможностью совершенствования черенковских гамма-телескопов, которая потенциально может быть реализована посредством применения УФ-фильтров, является повышение эффективности определения типа первичной частицы, так называемой „гамма-адронной сепарации“. Один из методов гамма-адронной сепарации предполагает измерение доли 
УФ-излучения в общем количестве черенковского излучения ШАЛ при фиксированном сигнале в основном диапазоне наблюдений (обычно $300-600 \mathrm{~nm}$ ). При таких условиях в излучении ШАЛ от протонов доля УФ-излучения в среднем выше, чем от гамма-квантов, что объясняется особенностями формирования и распространения ШАЛ в атмосфере Земли. Это обстоятельство и позволяет определять тип первичной частицы. Такой метод нашел практическое применение на телескопе ГТ-48 $[11,12]$, где для наблюдений в различных диапазонах длин волн были использованы различные типы ФЭУ (оптические и УФ), а также позднее дополнительно изучался теоретически в связи с планированием новых черенковских гамма-телескопов $[13,14]$. На черенковском гамма-телескопе TAIGA-IACT [15] с модернизированной камерой на SiPM тот же метод может быть реализован с помощью специальных фильтров с полосой пропускания в диапазоне $\lesssim 300 \mathrm{~nm}$, т. е., без применения двух различных типов SiPM. Это возможно потому, что эффективность детектирования некоторых типов SiPM в этом диапазоне все еще весьма значительна (5-20\%).

С целью отработки технологии создания SiPM-камер с возможностью использования съемных УФ-фильтров для планируемых наземных черенковских гамма-обсерваторий четвертого поколения (например, в проекте ALEGRO $[5,14,16])$ и повышения эффективности работы существующих черенковских гамма-обсерваторий в ФТИ им. А.Ф. Иоффе производится разработка детекторного кластера камеры для черенковского телескопа TAIGA-IACT (НИИЯФ МГУ, Иркутский университет). Планируется модернизация действующей камеры TAIGA-IACT посредством замены одного из детекторных кластеров на основе вакуумных ФЭУ ХР1911 на разрабатываемый в ФТИ детекторный кластер на основе SiPM OnSemi MicroFJ-60035. В рамках этого проекта производится количественное моделирование работы телескопа и, в частности, его детекторного блока с матрицей светочувствительных элементов.

Настоящая работа посвящена оценке параметров черенковского телескопа TAIGA-IACT с модернизированной камерой на SiPM, прежде всего пороговой энергии наблюдений при различных используемых фильтрах.

\section{1. Модель распространения ШАЛ и генерации черенковского излучения}

В настоящей работе использована полуаналитическая модель формирования и распространения ШАЛ, которая весьма детально описана в работе [14]. В рамках этой модели эволюция источников черенковского излучения (высокоэнергетических вторичных электронов и позитронов ШАЛ) описывается с помощью аналитических приближений [17-19] для средних продольных профилей распределения электронов ШАЛ (т.е. зависимостей количества электронов ШАЛ от высоты), а распростране-

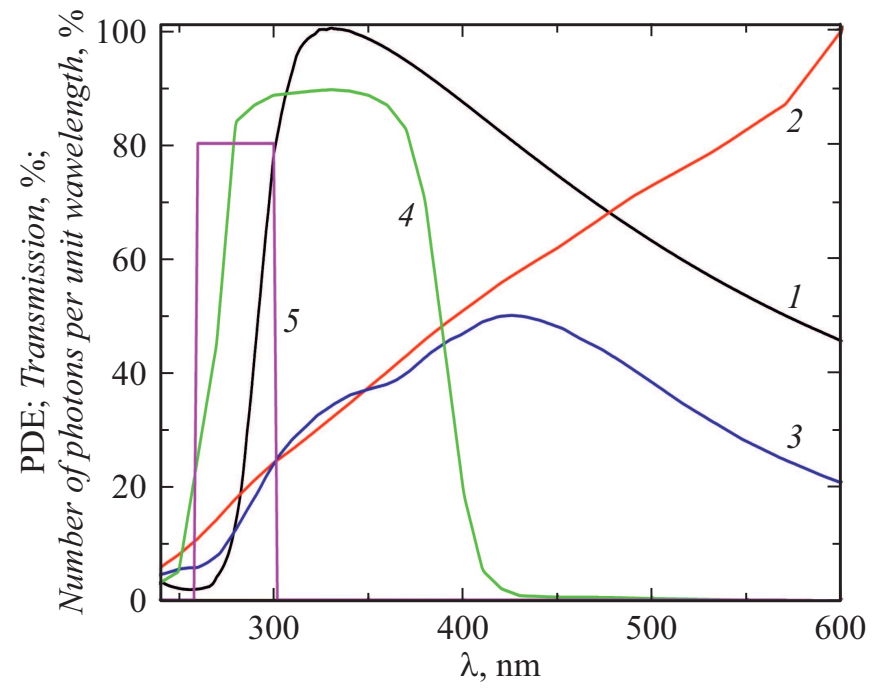

Рис. 1. Зависимости профилей от длины волны $\lambda: 1-$ типичный спектр черенковского излучения ШАЛ, нормированный на $100 \%$ в максимуме (на длине волны $326 \mathrm{~nm}$ для гаммакванта с энегией $1 \mathrm{TeV}), N_{\mathrm{Ch}}(\lambda) / N_{\mathrm{Ch}}(326 \mathrm{~nm}) ; 2$ - типичный спектр фона ночного неба по данным [27], нормированный на $100 \%$ на длине волны $600 \mathrm{~nm}, I_{\lambda} / I_{600} ; 3$ - эффективность детектирования SiPM-фотоумножителя OnSemi MicroFJ-60035 по данным [31], $\operatorname{PDE}(\lambda) ; 4-$ коэффициент передачи $F(\lambda)$ фильтра ZWB3 по данным [9]; $5-$ коэффициент передачи $F(\lambda)$ модельного MUV-фильтра 260-300 nm.

ние черенковского излучения ШАЛ - с помощью уравнения переноса излучения, которое решается численно.

Значительным отличием модели, использованной в настоящей работе, от модели [14] является модель атмосферы. В работе [14] была использована зависимость плотности атмосферы от глубины, справедливая в интервале значений высот от 1.8 до $36 \mathrm{~km}$ (ее отклонение от параметризации стандартной атмосферы, представленной в [20], составляет порядка $1 \%$ в указанном интервале высот). Однако на высотах ниже $1.8 \mathrm{~km}$ эта зависимость очень быстро становится совершенно неприменимой, поэтому в настоящей работе использована непосредственно параметризация Линсли стандартной атмосферы [21] (так называемая „Linsley model of 5 layers“, параметры которой приведены, например, в работе [20]), справедливая для высот от $0 \mathrm{~m}$ над уровнем моря. Это отличие является важным, поскольку высота расположения телескопа TAIGA-IACT составляет $675 \mathrm{~m}$ над уровнем моря. Также одним из ключевых аспектов модели атмосферы является распределение озона по высоте, поскольку именно поглощение на атмосферном озоне является основным процессом, отвечающим за убыль черенковских фотонов ШАЛ в УФ-диапазоне. Именно этот процесс в совокупности с зависимостью скорости генерации черенковского излучения от длины волны $\left(\sim \lambda^{-2}\right)$, определяемой формулой Франка-Тамма, приводит к формированию типичного спектра черенковского излучения ШАЛ (см. рис. 1) с ха- 
рактерным широким максимумом в диапазоне длин волн $310-330$ нм (в зависимости от типа и энергии первичной частицы). В настоящей работе была использована иная зависимость концентрации атмосферного озона, чем в работе [14]. В работе [14] были использованы экспериментальные данные наблюдений над Найроби (т.е. для тропических широт), которые не могут сколько-нибудь удовлетворительно описать ни вертикальное распределение, ни общее содержание озона в атмосфере в средних широтах (где располагается TAIGA-IACT), поэтому в настоящей работе для описания вертикального распределения озона в атмосфере был использован профиль из модели Крюгера-Минзнера [22] (измерения [23] показывают, что этот профиль удовлетворительно описывает вертикальное распределение озона в зимние месяцы в Сибири), а общая нормировка (т.е., общее содержание озона - колонковая концентрация) составила $440 \mathrm{DU}$ (Dobson units, $1 \mathrm{DU} \simeq 2.69 \cdot 10^{16} \mathrm{~cm}^{-2}$ ), что справедливо для района Забайкалья в феврале (см., например, [24]), вместо $345 \mathrm{DU}$ согласно стандартной модели Крюгера-Минзнера [22]. Такая перенормировка является первым приближением в адаптации стандартного профиля к конкретным местным условиям, но только в средних широтах, где в принципе применим профиль вертикального распределения озона из [22]. Основанием для подобного приближения служат данные исследования [25], где было указано на корреляцию между общим содержанием озона и концентрацией озона в нижней стратосфере.

Корректность использованной модели была проверена посредством сравнения с результатами расчетов с использованием стандартного вычислительного пакета CORSIKA [20], предназначенного для моделирования ШАЛ. Поскольку пакет CORSIKA предназначен для многочастичного моделирования, он позволяет определять не только средние значения искомых величин, но и их распределения, и соответственно параметры этих распределений, в частности, средние квадратичные отклонения исследуемых величин. Расчеты показывают, что общее количество черенковских фотонов ШАЛ от протонов, оцененное по модели, используемой в настоящей работе, и соответствующая величина, оцененная с помощью CORSIKA, различаются менее чем на $1 \sigma$ в диапазоне энергий от 1 до $30 \mathrm{TeV}$ как при учете поглощения атмосферным озоном, так и при отсутствии последнего. Кроме того, было произведено сравнение с результатом работы [26], использующей подход, промежуточный между полуаналитической моделью [14] и многочастичными расчетами: продольные профили рассчитывалась с помощью аналитических приближений, но функция распределения вторичных электронов ШАЛ по энергии рассчитывалась методом Монте-Карло. Результат работы [26] - среднее количество черенковских фотонов $2.6 \cdot 10^{15}$ в диапазоне длин волн $300-450 \mathrm{~nm}$ от первичного протона с энергией $10^{8} \mathrm{TeV}$ без учета поглощения атмосферным озоном - был воспроизведен с точностью $5 \%$, что также меньше отклонения $1 \sigma$, определенного в работе [26]. Отличие может объясняться разницей использованных вычислительных методов. Таким образом, модель генерации и распространения электронов и черенковского излучения ШАЛ, использованная в настоящей работе, показывает удовлетворительные результаты по вычислению общего количества черенковских фотонов ШАЛ (при сопоставлении с результатами других широко признанных моделей) в довольно широком диапазоне энергий (как минимум, $\left.1-10^{8} \mathrm{TeV}\right)$

\section{2. Фон ночного неба}

Функциональная зависимость интенсивности фонового излучения $I_{\lambda}$ от длины волны $\lambda$ (т.е., спектральный профиль фона ночного неба, (рис. 1) была получена на основании данных из работы [27]. Общая нормировка составила $I=\int I_{\lambda} d \lambda=3 \cdot 10^{12}$ photon $\cdot \mathrm{m}^{-2} \mathrm{~s}^{-1}$ ster $^{-1}$ в диапазоне длин волн 300-600 nm на основании данных, предоставленных группой TAIGA-IACT [15], что также хорошо согласуется с данными работы [28] для широкоугольного детектора с полем зрения $\gg 1^{\circ}$, размещенного в средних широтах, при осуществлении наблюдений в направлении зенита и вне галактической плоскости. ${ }^{2}$

\section{3. Модель формирования сигнала в детекторной камере телескопа TAIGA-IACT}

В настоящей работе используется относительно простая модель телескопа TAIGA-IACT, допускающая аналитическую оценку (решение, выраженное в квадратурах) при известных значениях параметров падающего в телескоп излучения. Тем не менее она позволяет учесть влияние на величину сигнала всех ключевых узлов и конструкций оптического тракта телескопа: зеркала, фильтров, конусов Уинстона и детекторной камеры.

\section{1. Величина полезного сигнала в детекторной камере}

Величина полезного сигнала в детекторной камере черенковского гамма-телескопа TAIGA-IACT может быть оценена по следующей формуле:

$$
\begin{aligned}
& Q_{F}^{S}=\int_{0}^{\infty} D_{C h}(\lambda, r, E) V_{m}(\lambda) S_{m}\left(\frac{2 \sqrt{3} \Theta_{p x}^{2}}{\pi \Theta_{E A S}^{2}}\right) \\
& \times V_{W}(\lambda) \operatorname{PDE}(\lambda) F(\lambda) d \lambda,
\end{aligned}
$$

где $Q_{F}^{S}$ [ph.e./frame] - среднее количество фотоэлектронов на детекторный пиксель за стандартную экспо-

\footnotetext{
${ }^{2}$ Отметим, что несмотря на использование терминов „интенсивность“ и обозначений $I_{\lambda}, I$, эти величины характеризуют именно потоки частиц (как это принято в работе [28]), а не полные энергии излученных фотонов, и в этом смысле отличаются от обозначений, принятых в работе[27].
} 
зицию (фрэйм) продолжительностью $\tau=10 \mathrm{~ns}$ (которая примерно совпадает с длительностью черенковской вспышки 10-15 ns) в засвеченом пятне в фокальной плоскости. Значение $Q_{F}^{S}$ усреднено по засвеченным пикселям и реализациям ШАЛ. Индекс , $S^{\text {“ }}$ означает „полезный сигнал“и в зависимости от типа первичной частицы, может принимать следующие значения: ,g“ для событий, вызванных гамма-квантами; „р“ для событий, вызванных протонами; „еff“ для событий, вызванных протонами с энергией $E_{\mathrm{eff}}$, определенной условием (9) (см. подробные пояснения в разд. 4). Индекс „F“ означает „фильтр“ и в зависимости от типа используемого фильтра может принимать следующие значения: „орt“ при отсутствии фильтра; „ZWB“ при использовании фильтра ZWB3 (рис. 1, кривая 4); „MUV“ при использовании модельного УФ-фильтра (рис. 1, кривая 5). Величина $D_{C h}(\lambda, r, E) \mathrm{m}^{-2} \mathrm{~nm}^{-1}$ - средняя спектральная поверхностная плотность черенковских фотонов ШАЛ от первичных частиц с энергией $E$ на расстоянии $r$ от оси ШАЛ на длине волны $\lambda$ (интегральный по фрэйму $\tau$ спектральный поток черенковских фотонов ШАЛ, т.е., количество черенковских фотонов ШАЛ на единицу площади поверхности наблюдения в единичном интервале длин волн), $V_{m}(\lambda)$ - коэффициент отражения зеркала, $S_{m} \simeq 9.6 \mathrm{~m}^{2}$ - площадь зеркала, $\Theta_{p x}=0.36^{\circ}[29,30]-$ угловой размер пикселя, ${ }^{3} \Theta_{E A S} \simeq 2.6^{\circ}$ - типичный угловой размер ШАЛ (который определяется углом раствора конуса черенковского излучения высокоэнергичных электронов в воздухе, см., например, [26]), $V_{W}(\lambda)-$ коэффициент передачи конуса Уинстона, $\operatorname{PDE}(\lambda)-$ эффективность детектирования фотоприемника $\mathrm{SiPM}$ на длине волны $\lambda[31]$ (рис. 1), $F(\lambda)$ - коэффициент пропускания используемого фильтра на длине волны $\lambda$ (рис. 1). Множитель $\left(2 \sqrt{3} \Theta_{p x}^{2}\right) /\left(\pi \Theta_{E A S}^{2}\right)$ имеет следующее происхождение: каждый пиксель соответствует определенному участку поля зрения телескопа (именно в этом смысле принято говорить об „угловом размере пикселя“), поэтому при регистрации каждого конкретного события засвечивается количество пикселей, которое определяется угловым размером наблюдаемого объекта, в рассматриваемом случае - угловым размером области генерации черенковского излучения ШАЛ. Соответственно количество засвеченных пикселей камеры примерно может быть оценено как $\simeq\left(\pi \Theta_{E A S}^{2}\right) /\left(2 \sqrt{3} \Theta_{p x}^{2}\right)$. Численный коэффициент $2 \sqrt{3}$ учитывает то обстоятельство, что пиксель имеет форму правильного шестиугольника, а не круга.

Для оценки величины регистрируемого сигнала $Q_{F}^{S}$ была выбрана величина спектральной поверхностной плотности на расстоянии $120 \mathrm{~m}$ от оси ШАЛ. Это сделано по следующим соображениям. С одной стороны, на расстояниях свыше 120-140 m поверхностная плотность убывает по закону $\sim r^{-2}$, где $r-$ расстояние до

\footnotetext{
${ }^{3}$ Не следует путать эту величину с полем зрения пикселя, которое составляет $35^{\circ}[15]$, и позволяет каждому пикселю „видеть“ все участки зеркала телескопа.
}

оси ШАЛ, что вместе с узкой направленностью ШАЛ является одной из главных причин ограничения эффективной площади детектирования черенковских телескопов на уровне $\sim 10^{5} \mathrm{~m}^{2}$. С другой стороны, значение $D_{C h}(\lambda, 120 \mathrm{~m}, E)$ близко к минимальному среднему (по реализациям ШАЛ) значению $D_{C h}$ в области $r \leq 120 \mathrm{~m}$ : на высоте расположения телескопа TAIGA-IACT (675 m над уровнем моря) для вертикально падающих ШАЛ с энергией первичной частицы $\lesssim 10 \mathrm{TeV}$ может происходить лишь небольшое $(\sim 10 \%)$ понижение среднего значения поверхностной плотности при приближении к оси ШАЛ, в то время как для ШАЛ от частиц более высоких энергий, наоборот, происходит значительный (на порядок) рост средней поверхностной плотности черенковских фотонов при приближении к оси ШАЛ (т.е. для таких ШАЛ с прицельным параметром ${ }^{4} \leq 120 \mathrm{~m}$ средний сигнал будет заведомо выше оценки). Таким образом, выбранное значение $D_{C h}(\lambda, 120 \mathrm{~m}, E)$ обеспечивает достаточно надежную оценку минимального значения среднего сигнала в области значений прицельных параметров $r \leq 120 \mathrm{~m}$, типичной для наблюдений ШАЛ черенковскими телескопами.

Величина $D_{C h}(\lambda, 120 \mathrm{~m}, E)$ по формуле

$$
D_{C h}(\lambda, 120 \mathrm{~m}, E)=\rho_{120} \frac{N_{C h}(\lambda, E)}{N_{\text {tot }}(1.6 \mathrm{TeV})},
$$

где $E-$ энергия первичной частицы, $\rho_{120} \mathrm{~m}^{-2}-$ средняя поверхностная плотность черенковских фотонов ШАЛ от первичных частиц с энергией $1.6 \mathrm{TeV}$ в исследуемом диапазоне $\left[\lambda_{1}=240 \mathrm{~nm} ; \lambda_{2}=600 \mathrm{~nm}\right]$ на расстоянии $120 \mathrm{~m}$ от оси ШАЛ. Референсные (опорные) значения $\rho_{120}=\rho(120 \mathrm{~m}, 1.6 \mathrm{TeV})$ составляют для протонов $\simeq 82 \mathrm{~m}^{-2}$, а для для гамма-квантов $\simeq 305 \mathrm{~m}^{-2}$. Эти значения получены непосредственной экстраполяцией данных работы [32], в которой расчет величины $\rho(r)$ был выполнен для условий месторасположения установки TUNKA (и телескопа TAIGA-IACT соответственно), диапазона длин волн [300 nm; $550 \mathrm{~nm}$ ] (в котором содержится примерно на $\simeq 20 \%$ меньше черенковских фотонов ШАЛ, чем в диапазоне $\left.\left[\lambda_{1} ; \lambda_{2}\right]\right)$ и энергии $1.6 \mathrm{TeV}$. Величина $N_{C h}(\lambda, E)\left[\mathrm{nm}^{-1}\right]-$ спектральная плотность (количество черенковских фотонов в пучке ШАЛ в единичном интервале длин волн) на длине волны $\lambda$, вычисленная согласно модели [14], $N_{t o t}(E)=\int N_{C h}(\lambda, E) d \lambda-$ полное количество черенковских фотонов ШАЛ в диапазоне $\left[\lambda_{1} ; \lambda_{2}\right]$. Значения $N_{t o t}(1.6 \mathrm{TeV})$ составляют для протонов $\simeq 3.54 \cdot 10^{7}$, а для гамма-квантов $\simeq 5.13 \cdot 10^{7}$.

В предположении слабой зависимости коэффициента отражения зеркала $V_{m}(\lambda)$ и коэффициента передачи конуса Уинстона $V_{W}(\lambda)$ от длины волны $\lambda$ в исследуемом диапазоне $\left[\lambda_{1} ; \lambda_{2}\right]$, эти функции в формуле (1) могут быть приближенно оценены их средними значениями $\bar{V}_{m}=0.8$ и $\bar{V}_{W}=0.75$. С учетом (2), формула (1) может

\footnotetext{
${ }^{4}$ В случае вертикального падения расстояние до оси ШАЛ совпадает с прицельным параметром первичной частицы.
} 


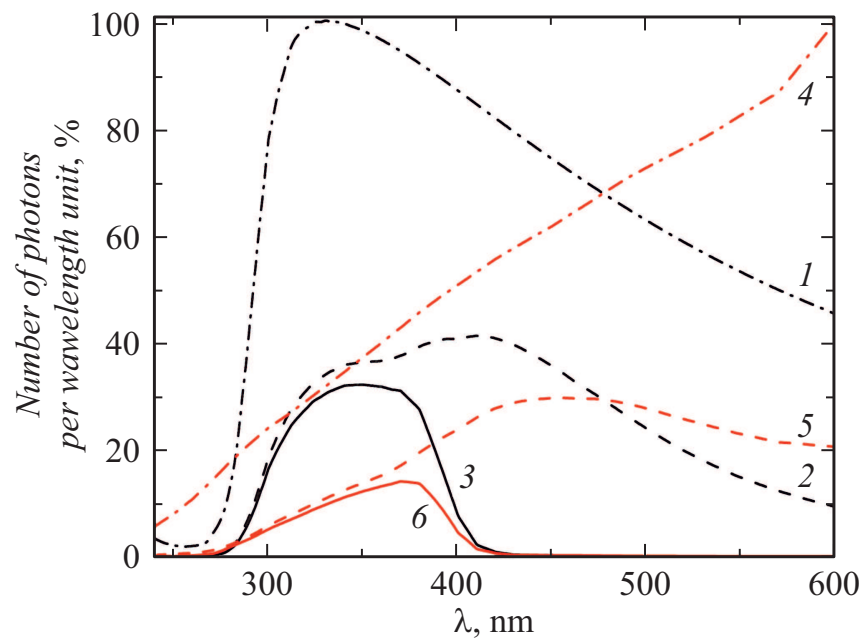

Рис. 2. Величины, определяющие скорость регистрации фотонов ШАЛ и фотонов фона ночного неба, в зависимости от длины волны $\lambda$ : 1 - профиль типичного спектра черенковского излучения ШАЛ, $N_{\mathrm{Ch}}(\lambda) / N_{\mathrm{C} h}(326 \mathrm{~nm}) ; 2$ - профиль скорости регистрации фотонов ШАЛ с помощью SiPMдетектора OnSemi MicroFJ-60035, $\operatorname{PDE}(\lambda) N_{\mathrm{Ch}}(\lambda) / N_{\mathrm{Ch}}(326 \mathrm{~nm})$; 3 - профиль скорости регистрации фотонов ШАЛ с помощью SiPM-детектора OnSemi MicroFJ-60035 при использовании фильтра ZWB3, $F(\lambda) \operatorname{PDE}(\lambda) N_{\mathrm{Ch}}(\lambda) / N_{\mathrm{Ch}}(326 \mathrm{~nm}) ; 4-$ профиль типичного спектра фона ночного неба по данным [27], $I_{\lambda} / I_{600} ; 5$ - профиль скорости регистрации фотонов фона ночного неба с помощью SiPM-детектора OnSemi MicroFJ-60035, $\operatorname{PDE}(\lambda) I_{\lambda} / I_{600} ; 6$ - профиль скорости регистрации фотонов фона ночного неба с помощью SiPM-детектоpa OnSemi MicroFJ-60035 при использовании фильтра ZWB3, $F(\lambda) \operatorname{PDE}(\lambda) I_{\lambda} / I_{600}$.

быть переписана в следующей форме:

$$
Q_{F}^{S} \simeq \rho_{120} \frac{N_{t o t}(E)}{N_{\text {tot }}(1.6 \mathrm{TeV})} \bar{V}_{m} S_{m}\left(\frac{2 \sqrt{3} \Theta_{p x}^{2}}{\pi \Theta_{E A S}^{2}}\right) \bar{V}_{W} T_{S}
$$

где $T_{S}$ - интегральный коэффициент (усредненный по фотонному распределению коэффициент детектирования с учетом действия фильтра), задаваемый формулой

$$
T_{S}=\int_{\lambda_{1}}^{\lambda_{2}} \frac{N_{C h}(\lambda, E)}{N_{t o t}(E)} \operatorname{PDE}(\lambda) F(\lambda) d \lambda .
$$

Пример функциональной зависимости подынтегрального выражения (4) как без использования фильтра, так и при использовании фильтра ZWB3 приведен на рис. 2.

Следует отметить, что максимальное значение полезного сигнала в области засвеченного пятна в фокальной плоскости может в 3-5 раз превышать среднее значение, но данный эффект не может быть учтен в рамках модели, используемой в настоящей работе, поскольку его рассмотрение требует многочастичного расчета (методом Монте-Карло). Тем не менее если в качестве триггера для регистрации полезного события используется превышение сигналом порогового значения в нескольких (например, трех) пикселях, то среднее значение вполне удовлетворительно подходит для описания ситуации, при которой должна происходить выработка триггера, и оценки соответствующей энергии первичной частицы ШАЛ (т.е. пороговой энергии наблюдений черенковского телескопа).

\section{2. Величина шумового сигнала}

В рамках тех же приближений, что были использованы в разд. 3.1, средняя величина шумового сигнала, вызванного фоном ночного неба, может быть оценена по следующим формулам:

$$
Q_{F}^{N} \simeq N_{p h} \bar{V}_{W} T_{N}
$$

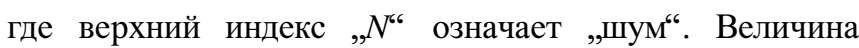
$N_{p h}$ - среднее количество „шумовых“ фотонов, падающих на входное окно конуса Уинстона, $T_{N}$ - коэффициент детектирования с учетом действия фильтра, усредненный по распределению „шумовых“ фотонов от длины волны $\lambda$. Величина $N_{p h}$ может быть вычислена по формуле

$$
N_{p h} \simeq I \tau \frac{\sqrt{3}}{2}\left(\Theta_{p x}\right)^{2} \bar{V}_{m} S_{m},
$$

где $I=\int I_{\lambda} d \lambda$ [photon $\left.\cdot \mathrm{m}^{-2} \mathrm{~s}^{-1} \mathrm{ster}^{-1}\right]$ - интегральная интенсивность фона ночного неба в исследуемом диапазоне. Коэффициент $T_{N}$ дается следующей формулой:

$$
T_{N}=\int_{\lambda_{1}}^{\lambda_{2}} \frac{I_{\lambda}}{I} \operatorname{PDE}(\lambda) F(\lambda) d \lambda .
$$

Функциональная зависимость подынтегрального выражения (7) как без использования фильтра, так и при использовании фильтра ZWB3 приведена на рис. 2.

Реальное (мгновенное) количество „шумовых“ фотонов, падающих на входное окно конуса Уинстона является случайной величиной, распределенной по закону Пуассона. Таким образом, среднеквадратичное отклонение этой величины может быть оценено как $\sigma_{p h}=\sqrt{N_{p h}}$, а среднеквадратичное отклонение величины шумового сигнала соответственно как: ${ }^{5}$

$$
\sigma_{F}^{N} \simeq \bar{V}_{W} T_{N} \sqrt{N_{p h}} .
$$

Знания величин $Q_{F}^{N}$ и $\sigma_{F}^{N}$ достаточно для проведения оценки пороговой энергии наблюдений черенковского телескопа.

\section{4. Результаты моделирования и обсуждение}

Основные результаты модельных расчетов представлены на рис. 3-5. На рис. 3 представлены зависимости

\footnotetext{
${ }^{5}$ Среднеквадратичными отклонениями величин $V_{m}(\lambda), \quad V_{W}(\lambda)$, $\operatorname{PDE}(\lambda)$ и $F(\lambda)$ при проведении оценки (8) мы пренебрегаем.
} 


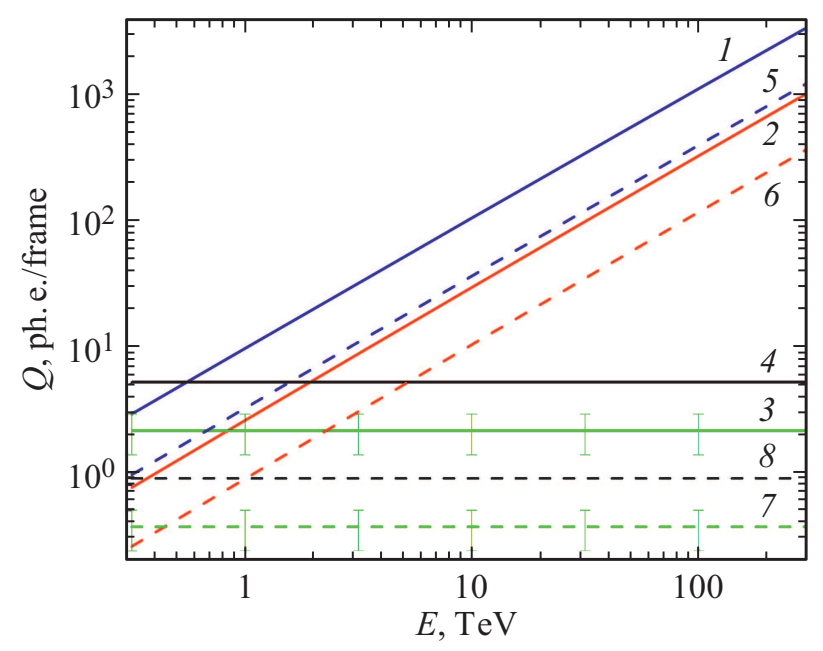

Рис. 3. Зависимости среднего полезного и шумового сигналов от энергии первичной частицы: 1 - средний полезный сигнал $Q_{\mathrm{opt}}^{g}$ от событий, вызванных гамма-квантами, при регистрации без фильтра; $2-$ средний полезный сигнал $Q_{\text {opt }}^{p}$ от событий, вызванных протонами, при регистрации без фильтра; 3 шумовой сигнал $Q_{\mathrm{opt}}^{N} \pm \sigma_{\text {opt }}^{N}$, вызванный фоном ночного неба, при регистрации без фильтра; $4-$ пороговое значение $Q_{\mathrm{opt}}^{\text {th }}$ для записи сигнала при регистрации без фильтра; 5 средний полезный сигнал $Q_{Z W B}^{g}$ от событий, вызванных гаммаквантами, при регистрации с использованием фильтра ZWB3; 6 - средний полезный сигнал $Q_{Z W B}^{p}$ от событий, вызванных протонами, при регистрации с использованием фильтра ZWB3; 7 - шумовой сигнал $Q_{Z W B}^{N} \pm \sigma_{Z W B}^{N}$, вызванный фоном ночного неба, при регистрации с использованием фильтра ZWB3; 8 пороговое значение $Q_{Z W B}^{t h}$ для записи сигнала при регистрации с использованием фильтра ZWB3 (см. пояснение в тексте).

средней величины полезного сигнала от энергии для различных типов первичных частиц при регистрации как без использования УФ-фильтра (сплошные кривые), так и при использовании фильтра ZWB3 (штриховые кривые). Кроме того, на рис. 3 представлены результаты расчетов шумового сигнала (его среднего значения и среднеквадратичного отклонения), а также приведено значение порога регистрации $Q_{F}^{t h}=Q_{F}^{N}+4 \sigma_{F}^{N}$. Такая величина порогового значения обеспечивает достаточно низкую частоту ложных срабатываний триггера регистрации сигнала.

Уровень шума, обусловленный фоном ночного неба, в камере телескопа без использования фильтров составляет $2.14 \pm 0.76$ ph.e./frame (что соответствует $Q_{o p t}^{t h}=5.19$ ph.e./frame), а при использовании фильтра ZWB3 $0.37 \pm 0.13$ ph.e./frame (что соответствует $Q_{Z W B}^{t h}=0.9$ ph.e./frame). Таким образом, представленные данные позволяют сделать вывод о том, что использование фильтра ZWB3 позволяет подавить шум в камере примерно в 5.8 раза, в то время как полезный сигнал подавляется примерно в 2.9 раза. Таким образом, при рассматриваемых спектральных профилях (рис. 1,2), шум подавляется фильтром сильнее, чем полезный сигнал. Вместе с тем обстоятельством, что значение среднего сигнала больше $\simeq 1$ ph.e./frame для гамма-квантов при $E \gtrsim 0.3 \mathrm{TeV}$ и для протонов при $E \gtrsim 1 \mathrm{TeV}$ (т. е. имеется достаточное количество информации для проведения морфологического количественного анализа изображения регистрируемой черенковской вспышки ШАЛ), это приводит к следующему нетривиальному следствию: пороговая энергия наблюдений телескопа TAIGA-IACT с камерой на SiPM может не увеличиться при применении УФ-фильтра, а наоборот, уменьшиться. Насколько известно авторам, подобный эффект ранее не упоминался в литературе, в том числе, не наблюдался на черенковских телескопах, где применялись УФ-фильтры [7-9] и не предсказывался в рамках численного моделирования. По всей видимости это связано с тем, что при применении фильтров величина полезного сигнала падала настолько сильно, что, несмотря на эффективное подавление шума, на типичных пороговых энергиях полезный сигнал сам по себе уже нес недостаточное для адекватной обработки количество информации о событии.

Отношение величин $Q_{F}^{S}$ и $Q_{F}^{\text {th }}$ (пересечения кривых $Q_{F}^{S}$ и $\left.Q_{F}^{t h}\right)$ позволяет явно оценить значения пороговой энергии для наблюдений различных частиц: при осуществлении наблюдения без фильтра эта величина составляет $\simeq 0.6 \mathrm{TeV}$ для гамма-квантов и $\simeq 2 \mathrm{TeV}$ для протонов, а при осуществлении наблюдения с использованием фильтра ZWB3 - $\simeq 0.3 \mathrm{TeV}$ для гамма-квантов и $\simeq 1 \mathrm{TeV}$ для протонов. Снижение пороговой энергии наблюдений при использовании фильтра является хотя и неожиданным, но полезным эффектом для работы черенковского телескопа, поскольку позволяет значительно увеличить количество регистрируемых высокоэнергичных гамма-квантов от космических источников (так как типичные спектры потоков гамма-излучения от таких объектов имеют степенной характер $\sim E^{-\alpha}$ с показателем $\alpha=2.5-3.5$, т. е. при уменьшении энергии наблюдений в 2-3 раза, поток гамма-излучения от наблюдаемого источника возрастает на порядок). Однако следует иметь ввиду, что скорость счета, обусловленного протонами космических лучей, также значительно возрастает (так как спектр космических лучей также имеет степенной характер $\sim E^{-2.7}$ в рассматриваемом диапазоне энергий), а протонные события являются одной из составляющих „шума“ для черенковских телескопов, нацеленных на наблюдение космического гамма-излучения. Таким образом, снижение пороговой энергии наблюдений требует сохранения возможностей для гамма-адронной сепарации наблюдаемых событий. В рассматриваемой ситуации такие возможности, по всей видимости, есть, поскольку, как упоминалось выше, количество информации в полезном сигнале от частиц с пороговой энергией достаточно для проведения эффективного морфологического анализа изображений черенковских вспышек ШАЛ в телескопе, который является основой гамма-адронной сепарации в современных черенковских телескопах. Однако более точный ответ на этот вопрос требует проведения многочастичного 
моделирования черенковского излучения ШАЛ и его взаимодействия с оптическими системами черенковского телескопа методом Монте-Карло.

Следует также отметить следующее обстоятельство: прогнозируемое значение пороговой энергии наблюдений черенковского телескопа TAIGA-IACT с модернизированной камерой на $\mathrm{SiPM}(\simeq 0.6 \mathrm{TeV})$ примерно в 2.5 раза ниже, чем значение пороговой энергии текущей конфигурации TAIGA-IACT с камерой на вакуумных фотоумножиелях $(\simeq 1.5 \mathrm{TeV}$ по данным группы TAIGA-IACT [15]). Это объясняется более высоким (примерно в 2 раза) значением эффективности детектирования SiPM OnSemi MicroFJ-60035 (максимум PDE составляет примерно $50 \%$ на длине волны $\simeq 425 \mathrm{~nm}$, ширина $\mathrm{PDE}$ на полувысоте составляет $\simeq 265 \mathrm{~nm}$ при напряжении смещения $6 \mathrm{~V}$ [31]), чем у вакуумных фотоумножиелей XP1911 (максимум PDE составляет примерно $26.5 \%$ на длине волны $\simeq 380 \mathrm{~nm}$, ширина PDE на полувысоте составляет $\simeq 190 \mathrm{~nm}$ согласно данным, предоставленным группой TAIGA-IACT [15]).

Данные, приведенные на рис. 3, также позволяют получить представление о максимальных значениях сигналов, которые будет регистрировать телескоп в диапазоне энергий до $300 \mathrm{TeV}$. Значение среднего сигнала от гамма-квантов с энергией $300 \mathrm{TeV}$ (максимального в исследуемом диапазоне энергий) составляет около 3400 ph.e./frame, что позволяет оценить величину максимального сигнала в отдельном наиболее ярко засвеченном пикселе на уровне около $2 \cdot 10^{4}$ ph.e./frame. В планируемой конфигурации субблока камеры на SiPM [33] предполагается, что пиксель будет состоять из более чем 70 тысяч активных микроячеек, каждая из которых в состоянии эффективно зарегистрировать один фотон в течение $150 \mathrm{~ns}$ (время релаксации микроячейки). Такое соотношение количества активных микроячеек и ожидаемой максимальной загрузки практически гарантирует, что в процессе работы в ночные часы не произойдет насыщения детекторов и просчета фотонов полезного сигнала.

Помимо подавления шума, вызванного в камере телескопа оптическим фоном ночного неба, еще одним важным аспектом применения УФ-фильтров в черенковских телескопах является возможность повышения эффективности гамма-адронной сепарации [11-14]. Метод гамма-адронной сепарации с использованием УФ-компоненты черенковского излучения ШАЛ основывается на следующем свойстве ШАЛ: при фиксированном значении поверхностной плотности черенковских фотонов ШАЛ доля УФ-излучения в диапазоне $\leq 300 \mathrm{~nm}$ по отношению к количеству излучения в полном исследуемом диапазоне $\left[\lambda_{1}, \lambda_{2}\right]$ оказывается больше в случае, если первичной частицей ШАЛ является протон, чем в случае, если первичной частицей ШАЛ является гамма-квант. Это связано с тем, что генерация черенковского излучения ШАЛ от протонов в среднем происходит глубже в атмосфере (т.е. на меньших высотах), чем в случае ШАЛ от гамма-квантов, поэтому черенковские фотоны ШАЛ от протонов проходят меньшую оптическую толщу по поглощению на атмосферном озоне, чем черенковские фотоны ШАЛ от гамма-квантов (этот эффект подробно рассмотрен в работе [14]). Для практической реализации этого метода можно использовать УФ-фильтр с полосой пропускания в диапазоне длин волн $\leq 300 \mathrm{~nm}$ для половины равномерно распределенных в фокальной плоскости телескопа пикселей детекторной камеры, а после регистрации события осуществлять сравнение сигналов от пикселей УФ и полного диапазона. Отношение этих сигналов будет зависеть от типа первичной частицы. В настоящее время не определен тип фильтра для диапазона $\leq 300 \mathrm{~nm}$, который можно было бы использовать для решения такой задачи. Имеющиеся в широком доступе фильтры обладают не вполне удовлетворительными характеристиками (например, слишком низким коэффициентом передачи, слишком узкой полосой пропускания или сложной геометрической формой и большими размерами, что конструктивно не позволяет включить их в состав детекторного кластера TAIGA-IACT), поэтому в настоящей работе для расчетов используется модельный профиль - коэффициент передачи гипотетического прямоугольного фильтра с пропускной способностью $80 \%$ в полосе длин волн 260-300 nm (см. рис. 1), который далее будет обозначен как MUV-фильтр (т.е. „model/middle UV-фильтр“). Ряд исследований показывает, что создание реального фильтра с подобными характеристиками, подходящего для практической реализации вышеописанного метода гамма-адронной сепарации вполне возможно [34] и является лишь вопросом стоимости.

На рис. 4 результаты расчетов средних значений полезного сигнала от ШАЛ, вызванных космическими частицами различных типов, и параметров шумового сигнала, вызванного оптическим фоном ночного неба, при использовании описанного выше MUV-фильтра. Кроме величины полезного сигнала от гамма-квантов и протонов, на рис. 4 представлена зависимость среднего полезного сигнала от протонов с энергией $E_{\mathrm{eff}}$ (т.е. функция $\left.Q_{\mathrm{o} p t}^{\mathrm{eff}}(E)=Q_{\mathrm{opt}}^{p}\left(E_{\mathrm{eff}}(E)\right)\right)-$ кривая 3 . Величина эффективной энергии $E_{\mathrm{eff}}(E)$ определяется исходя из следующего условия: сигнал $Q_{\mathrm{opt}}^{g}$ от гамма-квантов с энергией $E$ без использования фильтра и сигнал $Q_{\mathrm{opt}}^{p}$ от протонов с энергией $E_{\text {eff }}$ должны иметь одинаковые значения, то есть

$$
Q_{\mathrm{opt}}^{g}(E)=Q_{\mathrm{opt}}^{p}\left(E_{\mathrm{eff}}\right) .
$$

Расчеты показывают, что величина $E_{\text {eff }}$ может быть в явной форме определена по следующей формуле:

$$
E_{\mathrm{eff}}=B E_{0}\left(E / E_{0}\right)^{1-\beta},
$$

где $B=3.46, E_{0}=1 \mathrm{TeV}, \beta=0.013$. При использовании формулы $(10)$ величины $Q_{\mathrm{opt}}^{g}(E)$ и $Q_{\mathrm{opt}}^{\mathrm{eff}}(E)$ совпадают с точностью лучше $0.5 \%$ в диапазоне энергий первичных частиц $[0.3 ; 300] \mathrm{TeV}$. 


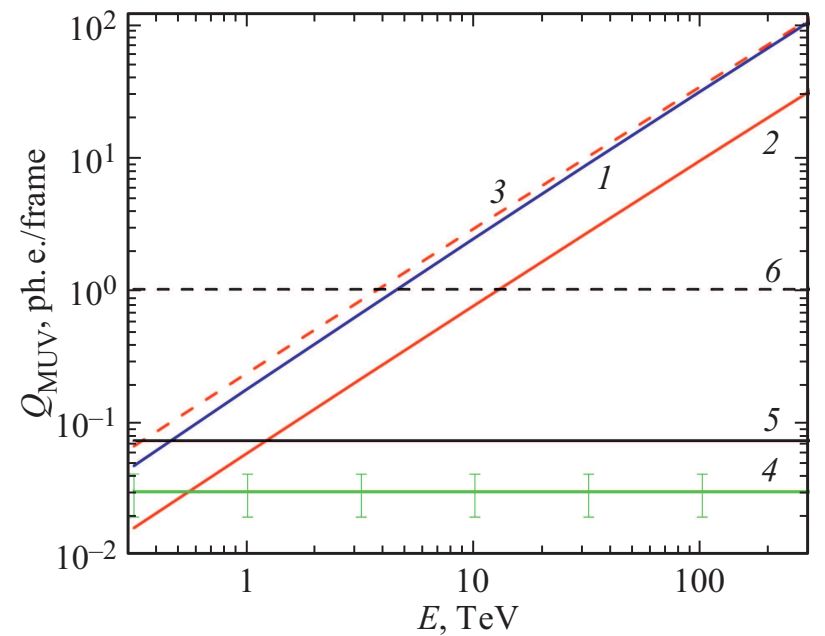

Рис. 4. Зависимости среднего полезного и шумового сигналов от энергии первичной частицы, а также пороговое значение для выдачи триггера на запись сигнала при использовании модельного MUV-фильтра 260-300 nm: 1 - средний полезный сигнал, $Q_{\mathrm{MUV}}^{g}$, от событий, вызванных гамма-квантами; 2 - средний полезный сигнал, $Q_{\mathrm{MUV}}^{p}$, от событий, вызванных протонами; $3-$ средний полезный сигнал, $Q_{\mathrm{MUV}}^{\mathrm{eff}}$, от событий, вызванных протонами с энергией $E_{\mathrm{eff}}$ (см. пояснение в тексте); 4 - шумовой сигнал, $Q_{\mathrm{MUV}}^{N} \pm \sigma_{\mathrm{MUV}}^{N}$, вызванный фоном ночного неба; $5-$ пороговое значение $Q_{\mathrm{MUV}}^{\text {th }}$ для записи сигнала (см. пояснение в тексте); $6-$ уровень единичного среднего сигнала 1 ph.e./frame.

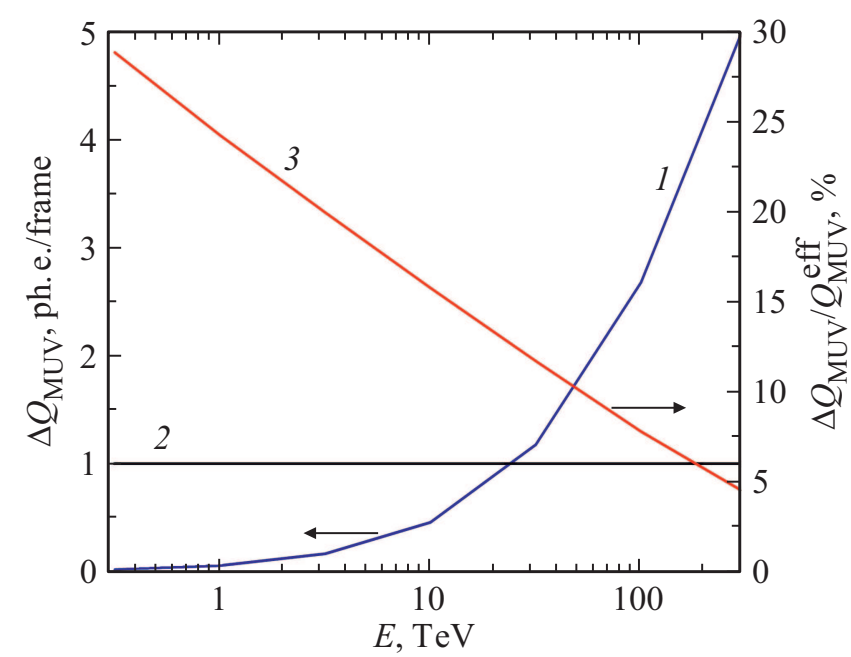

Рис. 5. Разности полезных сигналов от событий, вызванных гамма-квантами с энергией $E$ и протонами с энергией $E_{\mathrm{eff}}$ (см. пояснение в тексте) при использовании гипотетического прямоугольного MUV-фильтра 260-300 nm: 1 - разность абсолютных значений полезных сигналов, $\Delta Q_{\text {MUV }} ; 2-$ единичный уровень 1 ph.e./frame со значениями, отложенными на левой верикальной оси; 3 - относительная разность значений полезных сигналов $\Delta Q_{\mathrm{MUV}} / Q_{\mathrm{MUV}}^{\mathrm{eff}}$ со значениями, отложенными на правой верикальной оси.

Представленные расчетные данные позволяют сделать следующий вывод: шумовой сигнал подавляется до уров- ня $0.030 \pm 0.011$ ph.e./frame, что соответствует пороговому значению $Q_{\mathrm{MUV}}^{t h}=0.072$ ph.e./frame. Таким образом, при использовании фильтра со спектральными характеристиками, подобными характеристикам модельного MUV-фильтра, шумовой сигнал, вызванный оптическим фоном ночного неба в камере телескопа становится пренебрежимо малым. При таком низком значении шума пороговая энергия наблюдений будет определяться уже не условием $Q_{\mathrm{MUV}}^{S}=Q_{\mathrm{MUV}}^{t h}$, а скорее, наличием в самом исходном сигнале достаточного для корректного анализа параметров первичной частицы количества информации, что может определяться условием $Q_{\mathrm{MUV}}^{S} \geq 1$ (и тогда пороговая энергия может быть определена из условия $\left.Q_{\mathrm{MUV}}^{S}=1\right)$. Это приводит к следующим значениям пороговой энергии наблюдений при использовании модельного MUV-фильтра: $\simeq 4.6 \mathrm{TeV}$ для гамма-квантов и $\simeq 12.8 \mathrm{TeV}$ для протонов.

Максимальная в диапазоне энергий $[0.3 ; 300] \mathrm{TeV}$ величина среднего сигнала при использовании модельного MUV-фильтра не превышает 120 ph.e./frame, и, следовательно, можно ожидать, что максимальная величина сигнала (сигнал в наиболее сильно засвеченном пикселе) не будет превышать уровень около $10^{3}$ ph.e./frame. Таким образом, в детекторных пикселях, закрытых модельным фильтром, можно ожидать относительно невысокого уровня загрузки светочувствительных элементов и цепей системы считывания сигнала.

Наличие красной штриховой кривой 3 на рис. 4 позволяет увидеть разность между значениями среднего полезного сигнала в УФ-диапазоне $Q_{\mathrm{MUV}}^{\mathrm{eff}}(E)$ от протонов с энергией $E_{\mathrm{eff}}$ и сигнала $Q_{\mathrm{MUV}}^{g}(E)$ от гаммаквантов с энергией $E$ (т. е. при выполнении условия (9)). Видно, что эта разность значительно превышает $0.5 \%$ (точность выполнения условия (9)), таким образом, эта разность вызвана физическим эффектом, а не техническим дефектом моделирования. Тем не менее такая разность сигналов недостаточно велика, чтобы наглядно представлять результат на рис. 4, который построен в логарифмическом масштабе. В более наглядной форме разность сигналов представлена на рис. 5. При изменении энергии первичной частицы в диапазоне от 0.3 до $300 \mathrm{TeV}$ абсолютная разница $\Delta Q_{\mathrm{MUV}}=\left(Q_{\mathrm{MUV}}^{\mathrm{eff}}-Q_{\mathrm{MUV}}^{g}\right)$ значений среднего сигнала в маскированных пикселях от протонов с энергией $E_{\mathrm{eff}}$ и гамма-квантов с энергией $E$ меняется от 0 до 5 ph.e./frame. При этом относительная разница $\Delta Q_{\mathrm{MUV}} / Q_{\mathrm{MUV}}^{\mathrm{eff}}=\left(1-Q_{\mathrm{MUV}}^{g} / Q_{\mathrm{MUV}}^{\mathrm{eff}}\right)$ меняется от 29 до $4.5 \%$. Очевидно, что при малых значениях абсолютной разности использовать эту величину для гамма-адронной сепарации (или для повышения ее эффективности) практически невозможно. Использование этой величины возможно при условии $\Delta Q_{\mathrm{MUV}} \gtrsim 1$, что реализуется при энергии первичных гамма-квантов $\gtrsim 24 \mathrm{TeV}$. C другой стороны, при малой относительной разности $\Delta Q_{\mathrm{MUV}} / Q_{\mathrm{MUV}}^{\mathrm{eff}}$ использование рассматриваемого метода также, очевидно, затруднено, так как реальная измеряемая величина $Q_{\mathrm{MUV}}^{S}$ будет иметь отклонения, обусловленные как ошибками измерения, так и 
природой процессов формирования и распространения черенковского излучения ШАЛ, и эти флуктуации могут оказаться более $5-10 \%$. Следует отметить, что отношение $Q_{\mathrm{MUV}}^{S} / Q_{\mathrm{opt}}^{S}$ может оказаться более устойчивым, поскольку количества фотонов в различных диапазонах черенковской вспышки хотя и являются случайными величинами, но физически связаны, между ними имеется очевидная положительная корреляция (т.е. величины $Q_{\mathrm{MUV}}^{S}$ и $Q_{\mathrm{opt}}^{S}$ флуктуируют до некоторой степени одновременно и соразмерно, что приводит устойчивому отношению этих величин). К сожалению, полноценная проверка устойчивости отношения $Q_{\mathrm{MUV}}^{S} / Q_{\mathrm{opt}}^{S}$ (т. е. оценка флуктуаций этой величины) не может быть выполнена в рамках модели, используемой в настоящей работе, a требует проведения многочастичного моделирования методом Монте-Карло, что будет являтся предметом дальнейших исследований. Тем не менее даже при значительных флуктуациях отношения $Q_{\mathrm{MUV}}^{S} / Q_{\mathrm{opt}}^{S}$ предлагаемый метод гамма-адронной сепарации может являться дополнительным методом, повышающим эффективность стандартного метода гамма-адронной сепарации посредством анализа морфологических признаков изображения вспышки черенковского излучения ШАЛ (анализа параметров Хилласа). Относительная разность сигналов превышает $10 \%$ при энергиях первичных гамма-квантов $\lesssim 52 \mathrm{TeV}$. Таким образом, можно ожидать, что метод гамма-адронной сепарации посредством измерения доли УФ-излучения в общем количестве черенковского излучения ШАЛ может быть эффективно применен на телескопе TAIGA-IACT с модернизированной камерой на $\mathrm{SiPM}$ и УФ-фильтрами в диапазоне энергий первичных гамма-квантов $24-52 \mathrm{TeV}$.

\section{Заключение}

Использование в телескопе TAIGA-IACT детекторной камеры, основанной на кремниевых фотоумножителях OnSemi MicroFJ-60035 вместо вакуумных ФЭУ ХР1911, позволит примерно в два с половиной раза снизить значение порога регистрации космических гамма-квантов: с $\simeq 1.5$ до $\simeq 0.6 \mathrm{TeV}$. Последующее применение сплошной маски-фильтра типа ZWB3 позволит еще значительнее снизить этот порог до уровня $\simeq 0.3 \mathrm{TeV}$ за счет снижения уровня шума, вызванного в камере телескопа фотонами фона ночного неба, примерно в 5.8 раза (в то время как уровень среднего полезного сигнала понизится примерно в 2.9 раза). Это также позволит существенно увеличить рабочий цикл телескопа за счет наблюдений в лунные ночи и в сумерках. Применение узкого MUV-фильтра с полосой пропускания 260-300 nm, маскирующего половину камеры таким образом, что пиксели с фильтром и пиксели без фильтра чередуются в шахматном порядке, может позволить повысить эффективность гамма-адронной сепарации в диапазоне энергий первичных гамма-квантов $24-52 \mathrm{TeV}$ за счет измерения дополнительного параметра - доли УФ-излучения в обшем черенковском излучении ШАЛ.

\section{Благодарности}

Авторы благодарят анонимного рецензента за полезные замечания, учет которых позволил повысить качество текста статьи.

\section{Финансирование работы}

Авторы благодарят Российский научный фонд (РНФ) за поддержку в рамках проекта 19-72-20045.

\section{Конфрликт интересов}

Авторы заявляют, что у них нет конфликта интересов.

\section{Список литературы}

[1] Hsu C.C., Dettlaff A., Fink D., Goebel F., Haberer W., Hose J., Maier R., Mirzoyan R., Pimpl W., Reimann O., Rudert A., Sawallisch P., Schlammer J., Schmidl S., Stipp A., Teshima M. The Camera of the MAGIC-II Telescope. In International Cosmic Ray Conference. 2008. Vol. 3. International Cosmic Ray Conference. P. 1511-1514.

[2] Gazda E., Nguyen T., Otte N., Richards G. // J. Instrument. 2006. Vol. 11. P. 11015.

[3] Giavitto Gianluca, Ashton Terry, Balzer Arnim, Berge David, Brun Francois, Chaminade Thomas, Delagnes Eric, Fontaine Gerard, Füußling Matthias, Giebels Berrie, Glicenstein Jean-Francois, Gräber Tobias, Hinton Jim, Jahnke Albert, Klepser Stefan, Kossatz Marko, Kretzschmann Axel, Lefranc Valentin, Leich Holger, Lüdecke Hartmut, Lypova Iryna, Manigot Pascal, Marandon Vincent, Moulin Emmanuel, de Naurois Mathieu, Nayman Patrick, Ohm Stefan, Penno Marek, Ross Duncan, Salek David, Schade Markus, Schwab Thomas, Simoni Rachel, Stegmann Christian, Steppa Constantin, Thornhill Julian, Toussnel Francois. // Nucl. Instrument. Method. Phys. Res. A. 2017. Vol. 876. P. 35-38.

[4] CTA Consortium and Rene A. Ong. The Cherenkov Telescope Array Science Goals and Current Status. In Europ. Phys. J. Web Conf. 2019. Vol. 209. P. 01038.

[5] Bykov A.M., Aharonian F.A., Krassilchtchikov A.M., Kholupenko E.E., Aruev P.N., Baiko D.A., Bogdanov A.A., Vasilyev G.I., Zabrodskii V.V., Troitsky S.V., Tuboltsev Y.V., Kozhberov A.A., Levenfish K.P., Chichagov Y.V. // J. Tech. Phys. 2017. Vol. 6. P. 819-836.

[6] Knoetig et al. In Proc. of the 33rd ICRC, Rio de Janeiro, id. 695, arXiv: 1307.6116

[7] Chantell M., Akerlof C.W., Buckley J., Carter-Lewis D.A., Cawley M.F., Connaughton V., Fegan D.J., Fleury P., Gaidos J., Hillas A.M., Lamb R.C., Pare E., Rose H.J, Rovero A.C., Sarazin X., Sembroski G., Schubnell M.S., Urban M., Weekes T.C., Wilson C. Gamma-Ray Observations in Moonlight with the Whipple Atmospheric Cherenkov Hybrid Camera. Intern. Cosmic Ray Conf. 1995. Vol 2. P. 544. 
[8] Griffin S. VERITAS Collaboration. VERITAS Observations under Bright Moonlight. In 34th International Cosmic Ray Conference (ICRC2015). 2015. Vol. 34. N 7. Intern. Cosmic Ray Conf. P. 989.

[9] Guberman D., Cortina J., García R., Herrera J., Manganaro M., Moralejo A., Rico J., Will M. MAGIC Collaboration. Using UV-pass filters for bright Moon observations with MAGIC. In 34th International Cosmic Ray Conference (ICRC2015). Intern. Cosmic Ray Conf. 2015. Vol. 34. N 7. P. 1237.

[10] Ahnen M.L., Ansoldi S., Antonelli L.A., Arcaro C., Babić, and 140 co authors. Performance of theMAGIC telescopes under moonlight. Astroparticle Physics. 2017. N 9. 94:29-41.

[11] Zyskin Y.L., Vladimirsky B.M., Neshpor Y.I., Stepanian A.A., Fomin V.P., Shitov V.G. The Investigation of Cerenkov Flashes in Ultraviolet Spectral Region. 1987. N 2. Intern. Cosmic Ray Conf.P. 342.

[12] Neshpor Y.I., Chalenko N.N., Stepanian A.A., Kalekin O.R., Jogolev N.A., Fomin V.P., Shitov V.G. BL Lac: A New Ultrahigh-Energy Gamma-Ray Source. 2017. N 4. Astronomy Reports. P. 249-254.

[13] Rahman M.A., Bhat P.N., Acharya B.S., Chitnis V.R., Majumdar P., Vishwanath P.R. Gamma Ray and Hadron generated Čerenkov Photon Spectra at Various Observation Altitudes. Experimental Astronomy. 2001. N 4. P. 113-131.

[14] Kholupenko E.E., Bykov A.M., Aharonyan F.A., Vasiliev G.I., Krassilchtchikov A.M., Aruev P.N., Zabrodskii V.V., Nikolaev A.V. Detection of UV Radiation from Extensive Air Showers: Prospects for Cherenkov Gamma-Ray Astronomy. Journal of Technical Physics. 2018. N 11. P. 1603-1614.

[15] Kuzmichev L.A., Astapov I.I., Bezyazeekov P.A., Boreyko V., Borodin A.N., BudnevN.M., Wischnewski R., Garmash A.Y., Gafarov A.R, Gorbunov N.V., Grebenyuk V.M., Gress O.A., Gress T.I., Grinyuk A.A., Grishin O.G., Dyachok A.N., Zagorodnikov A.V, Zurbanov V.L., Ivanova A.L., Kazarina Y.A., Kalmykov N.N., Karpov N.I., Kindin V.V., Kirilenko P.S., Kiryuhin S.N., Kozhin V.A., KokoulinR.P., Kompaniets K.G., Korosteleva E.E., Kravchenko E.A., Kunnas M., Chiavassa A, Lagutin A.A., Lenok V.V., Lubsandorzhiev B.K., Lubsandorzhiev N.B., Mirgazov R.R., Mirzoyan R., Monkhoev R.D., Nachtigall R., Osipova E.A., Panasyuk M.I., Pankov L.V., Pakhorukov A.L., Petrukhin A.A., Poleschuk V.A., Popesku M., Popova E.G., Porelli A., Postnikov E.B., Prosin V.V., Ptuskin V.S., Pushnin A.A., Raikin R.I., Rubtsov G.I., Sagan Ya.I., Samoliga V.S., Sveshnikova L.G., Semeney Yu.A., Sidorenkov A.Y., Silaev A.A., Silaev A.A., Skurikhin A.V., Slunecka M., Sokolov A.V., Sulakov V.P., Tabolenko V.A., Tarashansky B.A., Tkachev L.G., Tkachenko A.V., Tluczykont M., Fedorov O.L., Horns A.D., Spiering C., Elshoukrofy A.Sh.M., Yashin I.I. // Physics of Atomic Nuclei. 2018. N 7. Vol. 81. N 4. P. 497-507.

[16] Kholupenko E.E., Aruev P.N., Baiko D.A., Bogdanov A.A., Vasilyev G.I., Zabrodskii V.V., Krasilshchikov A.M., Tuboltsev Y.V., Chichagov Y.V. // Physics of Atomic Nuclei. 2016. Vol. 79. N 12. P. $1542-1545$.

[17] K. Greisen. Progress in Cosmic Ray Physics. Vol. 3. Amsterdam : North-Holland, 1956.

[18] Ilina N.P., Kalmykov N.N., Prosin V.V. // Sov. J. Nucl. Phys. 1992. Vol. 55. P. 1540-1547. [Yad. Fiz.55,2756(1992)]

[19] Linsley J. GIL: a formula for EAS longitudinal profiles. International Cosmic Ray Conference. 2001. Vol. 2. P. 502.
[20] Heck D., Knapp J., Capdevielle J.N., Schatz G., Thouw T. CORSIKA: a Monte Carlo code to simulate extensive air showers. Forschungszentrum Karlsruhe $\mathrm{GmbH}$, Karlsruhe (Germany). V + 90 p., TIB Hannover, D-30167 Hannover (Germany), February 1998.

[21] The 1976 Standard Atmosphere Above 86-km Altitude. NASA SP-398. NASA Special Publication. P. 398. 1976.

[22] Krueger A.J., Minzner R.A. A mid-latitude ozone model for the 1976 U.S. 1976. N 8. Standard Atmosphere. Vol. 81. N 24. P. $4477-4481$

[23] Невзоров А.А., Долгий С.И., Невзоров А.В., Романовский О.А., Гриднев Ю.В. Измерения вертикальной стратификации озона на Сибирской лидарной станции. In: Актуальные проблемы радиофизики. VII Междунар. научнопрактическая конф. Томск, 2017. № 9. С. 143.

[24] Sklyadneva T.K., Lomakina N.Y., Bedareva T.V. Spatiotemporal distributions of total water vapor and total ozone in continental regions of Siberia and in the Far East „,continent-ocean“ transition zone. Atmos. Ocean. Opt. 2013. Vol. 26. P. 214.

[25] Божсков Р.Д. // Метеорология и гидрология. 1969. C. $100-108$.

[26] Berat C., Bottai S., De Marco D., Moreggia S., Naumov D., Pallavicini M., Pesce R., Petrolini A., Stutz A., Taddei E., Thea A. // Astroparticle Physics. 2010. Vol. 5. P. 221-247.

[27] Leinert C., Bowyer S., Haikala L.K., Hanner M.S., Hauser M.G., Levasseur-Regourd A.C., Mann I., Mattila K., Reach W.T., Schlosser W., Staude H.J., Toller G.N., Weiland J.L., Weinberg J.L., Witt A.N. The 1997 reference of diffuse night sky brightness. Astronomy and Astrophysics Supplement. 1998. N 1. P. 1-99.

[28] Mirzoyan R.Lorenz E. Measurement of the night sky light background at La Palma. Internal report of the HEGRA collaboration. MPI-PhE. 1994. P. 94-35.

[29] Lubsandorzhiev N., Astapov I., Bezyazeekov P., Boreyko V., Borodin A., Brueckner M., Budnev N., Chiavassa A., Dyachok A., Fedorov O., Gafarov A., Garmash A., Gorbunov N., Grebenyuk V., Gress O., Gress T., Grishin O., Grinyuk A., Horns D., Igoshin A., IvanovaA., Kalmykov $N$., Kazarina $Y$., Kindin $V$., Kirilenko $P$., Kiryuhin S., Kokoulin R., Kompaniets K., Korosteleva E., Kozhin V., Kravchenko E., Kunnas M., Kuzmichev L., Lemeshev Y., Lenok V., Lubsandorzhiev B., Mirgazov R., Mirzoyan R., Monkhoev R., Nachtigall R., Osipova E., Pakhorukov A., Panasyuk M.I., Pankov L., Petrukhin A., Poleschuk V., Popescu E., Popova E., Porelli A., Postnikov E., Prosin V., Ptuskin V., Rjabov E., Rubtsov G., Pushnin A., Sagan Y., Sabirov B., Samoliga V., Semeney Y., Silaev A., Silaev(junior) A., Sidorenkov A., Skurikhin A., Slunecka V., Sokolov A.V., Spiering C., Sveshnikova L., Tabolenko V., Tarashansky B., Tkachenko A., Tkachev L., Tluczykont M., Wischnewski R., Zagorodnikov A., Zhurov D., Zurbanov V., Yashin I. Camera of the first TAIGA-IACT: construction and calibration. In 35th International Cosmic Ray Conference (ICRC2017). Vol. 301 of International Cosmic Ray Conference. 2017. N 1. P. 757. 
[30] Budnev N., Astapov I., Barbashina N., Barnyakov A., Bezyazeekov P., Bogdanov P., Boreyko V., Brückner M., Chiavassa A., Chvalaev O., Dyachok A., Epimakhov S., Fedorov O., Fedoseev E., Gafarov A., Gorbunov N., Grebenyuk V., Gress O., Gress T., Grinyuk A., Grishin O., Haungs A., Hiller R., Horns D., Huege T., Ivanova A., Karpov N., Kalmykov N., Kazarina Yu., Kirilenko P., Kirichkov N., Kiryuhin S., Kleifges M., Kokoulin R., Kompaniets K., Korosteleva E., Kostunin D., Kozhin V., Kravchenko E., Kunnas M., Kuzmichev L., Lenok V., Lubsandorzhiev V., Lubsandorzhiev $\quad N$., Mirgazov R., Mirzoyan R., Monkhoev R., Nachtigall R., Osipova E., PakhorukovA., Panasyuk M., Pankov L., Perevalov A., Petrukhin A., Pivovarov S., Platonov V., Poleschuk V., Popova E., Porelli A., Postnikov E., Prosin V., Ptuskin V., Pushnin A., Rubtsov G., Rybov E., Samoliga V., Saunkin A., Schröder R., Semeney Yu., Silaev A., Silaev A., Skurikhin A., Sokolov A., Spiering C., Sveshnikova L., Tabolenko V., Tarashchansky B., Tikhonov Yu., Tkachenko A., Tkachev L., Tluczykont M., Voronin D., Wischnewski R., Yashin I., Yurin K., Zagorodnikov A., Zirakashvili V., Zurbanov V. // Nuclear Instruments and Methods in Phys. Res. A. 2017. Vol. 845. P. 330-333.

[31] ON Semiconductor. J-Series SiPM Sensors. Silicon Photomultipliers (SiPM), High PDE and Timing Resolution Sensors in a TSV Package; https:/www.onsemi. com/pub/Collateral/MICROJ-SERIES-D.PDF, 2017.

[32] Rastegarzadeh G., Khoshabadi S. // Iranian J. Astronomy and Astrophysics. 2014. Vol. 1. P. 127.

[33] Bogdanov A.A., Kholupenko E.E., Tuboltsev Yu.V., Chichagov Yu.V. Modeling of SiPM Performance for Detection of Cherenkov Radiation from Extensive Air Showers in UV- and Visible Ranges for Application at the TAIGA-IACT Telescope. Latvian J. Phis. and Tech. Science. 2020. N 1. P. 13. DOI: $10.2478 /$ pts-2020-0002

[34] Acеев B.A. Спектральные фильтры, выделяющие ультрафиолет. Частное сообщение, 2019. 